\title{
EDITORIAL \\ Communicating cancer risk from radiation exposures: nuclear accidents, total body radiation and diagnostic procedures
}

Bone Marrow Transplantation (2013) 48, 2-3; doi:10.1038/ bmt.2012.90; published online 29 October 2012

Most people know very little about radiation, except that it is frightening, can cause cancer, and is best avoided whenever possible. When faced with a possible radiation exposure, like after the Chernobyl and Fukushima nuclear power facility accidents, they may consult their physician about such risks. This is especially likely if their physician is a hematologist, cancer specialist or expert in blood cell and bone marrow transplantation (BMT).

Interestingly, readers of the journal Bone Marrow Transplantation are in the unique position to address these issues when prescribing extraordinarily high doses of ionizing radiation, for example, when we give total body radiation or a radionuclide linked to a monoclonal antibody (mAb) for pretransplant conditioning. We are also likely to expose people to ionizing radiation by ordering $\mathrm{X}$-ray studies, computed tomography (CT) scans and diagnostic or therapeutic radio-isotope studies. In fact, about $80 \%$ of our annual exposure to man-made ionizing radiations $(3 \mathrm{mSv})$ comes from tests and procedures ordered by physicians. This is almost as much as our annual exposure from natural radiation $(3.5 \mathrm{mSv})$ from the cosmos and terrestrial sources.

Another way transplant experts get involved in radiobiology is via nuclear accident/radiation incident planning. There are now networks of transplant physicians and centers in the Europe and the United States ready to respond to events like the explosion of an improvised nuclear or radiological device or weapon (so-called dirty-bomb, IND or IRD), or an accident at a nuclear power facility. Although the focus of these efforts is medical, inevitably the public will involve personnel with questions about radiation-related cancer risks.

Although it is easy to understand why people would approach transplant experts to discuss their concerns about radiation, most of us, unfortunately, know very little about precisely estimating cancer risk after radiation exposures. Nor do most of us know the best format in which to express cancer-risk to non-scientists. In this editorial, we suggest how transplant experts, other physicians, health authorities and governments can best inform patients, families and the public about cancer risks from ionizing radiation, especially from accidents at nuclear power facilities. A more detailed discussion of several risk-expression tools is available elsewhere. ${ }^{1}$

Understandably, most people have substantial, albeit unrealistic, concerns about the impact of radiation exposure on their health. They are especially focused on the deceptively simple question: What is my risk of cancer? Other important questions, usually after preliminary and contradictory data are released by governments, scientific experts (real and imagined) and the media are: How certain are you of the risk estimate you are telling me? How does this risk compare to other risks in my life? and, Is there anything I can do to avoid or decrease my risk from radiation? (avoid physicians is an easy answer). People need this information for many reasons, but especially for informed decision-making about traveling to Japan, eating sushi in New York, having a CT scan or voting on a proposition for a new nuclear power facility in their country or having a BM transplant. People with cancer are a special group because of several considerations: (1) the benefit: risk ratio of diagnostic and therapeutic radiation exposures is entirely different that of the public; and (2) the risk is less voluntary; they may not want to opt out of a therapy or test.

Effective communication of the risk of cancer in persons exposed to radiation is challenging. Conventional approaches of presenting cancer risks are indirect. They rely on units of dose (like $\mathrm{mSv}$ ) or concentrations of a radioactive substance in the environment (like Bq). Information given in this fashion is unlikely to inform most people; worse, it can be misleading. It also fails to consider that radiation-related cancer risk is highly dependent on age at time of exposure, remaining lifespan, exposure to other cancer-causing agents (like smoking) and other variables not encompassed in the expression of dose. Exposing an 80-year-old person to a dose of radiation has an entirely different implication than exposing a 3-year-old child to the same dose.

The fundamental problem with relying on dose or a level of radioactivity to express cancer risk is that the dose is only an intermediate quantity between exposure and risk. For example, when dose is used to express risk, it is typically compared with a regulatory dose limit, doses associated with natural background radiations, doses from medical procedures (like X-rays and $\mathrm{CT}$ scans) and/or doses of the Japanese A-bomb survivors. The implication is that if the estimated dose is below the dose or concentration values used for these comparisons, there should be no cause for concern, and that the risk at these dose levels is acceptable. People are typically unconvinced by such arguments.

Is there a better way to express potential cancer-related hazards of radiation exposures to people? Yes. Some alternatives: (1) a person's life-time risk of cancer regardless of cause; (2) excess lifetime risk resulting only from the additional radiation exposure related to the accident; (3) future total and/or excess life-time cancer risk for persons exposed in the past (or who will be exposed soon) and who are currently free of cancer; and (4) total and/or excess numbers of cancers anticipated in an exposed population (like people evacuated from Fukushima) over their life time.

All of these risk estimates need to be quantified with regard to uncertainty, which arises from several sources. Perhaps the greatest uncertainty comes from our extrapolation of risks from high doses (like the A-bomb survivors) to settings like Chernobyl and Fukushima, where doses are substantially lower and where exposure is prolonged over time. Although some scientists argue that very low radiation doses do not increase cancer risk, we should assume any dose can cause cancer until proved otherwise. Recently reported data from the Radiation Effects Research Foundation (RERF) are consistent with a linear, no-threshold relationship between radiation dose and cancer risk even at the lowest doses extending through zero. ${ }^{2,3}$ As the A-bomb exposures are similar (but not identical, because of a small high-energy neutron component and other variables) to our use of acute high-dose total body radiation, data from RERF also allow us to estimate cancer risk after total body radiation.

The life-time risk of cancer incidence in otherwise healthy individuals from an acute total body radiation with a radiation dose of 1 Gy received at age 30 is 0.15 (95\% confidence interval 
$0.08,0.24)$ for males and 0.18 (95\% confidence interval $0.14,0.26$ ) for females. ${ }^{4}$ Individuals already affected by cancer may have somewhat lower risks (perhaps by a factor up to 2 or more), as they have a lower chance of surviving long enough to develop other cancers later in life. These substantial risks, of course, need to be balanced against potential benefit in persons to whom we consider giving 10-12 Gy total body radiation. However, it should probably be included in future decision-making processes, comparing transplants to alternative therapies. Markov decision processes are an example (for example, see Gale ${ }^{5}$ ).

Effective communication of cancer risk to the public also requires a vital but volatile quantity: trust. Communicating cancerrisk assessments to people by dose estimates without addressing the fundamental issue of the cancer risk associated with radiation exposure may lead to a perception of censorship of risk information. Such perceptions of censorship diminish trust, which, once lost, is difficult if not impossible to regain.

People have bona fide concerns about radiation-related cancer risks and deserve direct, credible and intelligible answers, including an expression of our uncertainty. The challenge is to place this risk into context so that it can be compared with voluntary and involuntary cancer risks in everyday life, such as smoking cigarettes, flying in a jet or going into a basement containing radon gas. In a transplant context, we need to compare the risk of total body radiation to our life-time cancer risk (about $42 \%$ in a male and even higher in someone who already has one cancer) and to the cancer risk of alternative therapies. Placing risk estimates into context helps people weigh the importance of a cancer-risk and decide whether a future exposure is acceptable. Equally important is the ability to compare cancer risk with potential alternatives and with potential benefits (like having a CT scan). It is especially important to acknowledge that the uncertainty in our risk estimates is based on our present knowledge, and that this uncertainty may change as our state of knowledge improves. We hope this proposal will be followed when governments and international organizations release estimates of cancer risk from nuclear and radiation accidents. These guidelines should also help transplant specialists explain to patients and the public what publicly released data mean. They may also be useful in explaining excess cancer risk from the radiation we commonly expose people to, such as total body radiation and diagnostic radiological tests.

\section{CONFLICT OF INTEREST}

RP Gale is a part-time employee of Celgene Corp.

\section{ACKNOWLEDGEMENTS}

RP Gale is Visiting Professor of Haematology, Section of Haematology, Division of Experimental Medicine, Department of Medicine, Imperial College, London, UK. F. Owen Hoffman is president of SENES Oak Ridge, Inc., Center for Risk Analysis, Oak Ridge, TN, USA.

RP Gale ${ }^{1}$ and FO Hoffman ${ }^{2}$

${ }^{1}$ Section of Haematology, Division of Experimental Medicine, Department of Medicine, Imperial College, London, UK and

${ }^{2}$ SENES Oak Ridge, Inc., Center for Risk Analysis, Oak Ridge, TN, USA E-mail: robertpetergale@aol.com

\section{REFERENCES}

1 Hoffman FO, Kocher DC, Apostoaei Al. Beyond dose assessment: using risk with full disclosure of uncertainty in public and scientific communication. Health Phys 2011; 101: $591-600$.

2 National Research Council. Health Risks from Exposure to Low Levels of lonizing Radiation: BEIR VII Phase 2. National Academy Press: Washington, DC, 2006.

3 Ozasa K, Shimizu Y, Suyama A, Kasagi F, Soda M, Grant EJ et al. Studies of the mortality of atomic bomb survivors, report 14, 1950-2003: an overview of cancer and non cancer diseases. Radiat Res 2012; 177: 229-243.

4 Berrington de González AM, Mahesh M, Kim KP, Bhargavan M, Lewis R, Mettler F et al. Projected cancer risks from computed tomographic scans performed in the United States in 2007. Arch Intern Med 2009; 169: 2071-2077.

5 Gale RP. Markov model of CLL transplants. Bone Marrow Transplant 2012; 47: $1145-1146$. 